\title{
The Relation between the Good-Walker and Triple-Regge Formalisms for Diffractive Excitation
}

\author{
Gösta Gustafson \\ Dept. of Astronomy and Theoretical Physics, Lund University, \\ Slvegatan 14A, Lund, Sweden \\ E-mail: gosta.gustafson@thep.lu.se
}

\begin{abstract}
In this note we analyse the relation between the triple-pomeron and GoodWalker formalisms for diffractive excitation in DIS and hadronic collisions. In both approaches gap events are interpreted as the shadow of absorption into inelastic channels. We here argue that the two formalisms are just different views of the same phenomenon. We first demonstrate how this relation works in a simple toy model, and then show how the relevant features of the toy model are also realized in real perturbative QCD.
\end{abstract}

KeYwords: Diffraction, Pomeron, Triple-Regge, Good-Walker. 


\section{Contents}

1. Introduction 1

2. The toy model 2

3. Triple-pomeron formalism 3

4. Good-Walker formalism

5. Relation to QCD 5

6. Discussion 6

7. Conclusion 7

\section{Introduction}

Events with rapidity gaps contribute of the order $10 \%$ in DIS at HERA [1, 2], and in $p \bar{p}$ collisions at the CERN $S p \bar{p} S$ collider [3, 4] and the Tevatron [5]. Such events have commonly been interpreted as the shadow of absorption into inelastic channels, in analogy with diffraction in optics. Two different formalisms are based on this analogy: the MuellerRegge approach [6, 7] and the Good-Walker formalism [8].

In the Regge formalism the absorption is represented by cut pomeron or reggeon diagrams. Regge diagrams, cut in between two exchanged pomerons or reggeons, represent elastic scattering, while the amplitude for diffractive excitation is determined by the $3 \rightarrow 3$ scattering process. At high energies multi-regge diagrams are important, where the relative contributions from cut and uncut diagrams are given by the AGK cutting rules [9]. Recent analyses within this formalism include work by Kaidalov and coworkers 10, Ostapchenko [11], the Durham [12, 13], and the Tel Aviv groups [14, 15, 16].

In the Good-Walker formalism the projectile is written as a coherent sum of components, which are eigenstates to the interaction with the target. If the interaction strengths are different for the different components, the diffractive state no longer corresponds to the incoming coherent sum, and the cross section for diffractive excitation is determined by the fluctuations in the interaction strengths. In the analyses mentioned above, this formalism was used to describe low mass diffraction, but it has also been proposed to describe the full spectrum of excitation masses in the early work by Miettinnen and Pumplin [17], and more recently by Hatta et al. [18], and by the Lund group [19, 20, 21]. 
The aim of the present note is to argue that, although apparently quite different, the triple-pomeron and Good-Walker formalisms are just different views of the same phenomenon. Besides the common basis in the optical theorem, a common underlying dynamics for the two formalisms is suggested by the connection between the fluctuations in the cascade evolution and the triple-pomeron vertex, which is demonstrated in ref. [22]. Within the dipole cascade formalism it is there shown that both these effects are determined by the splitting of a dipole into two connected dipoles, sharing a common gluon (see also ref. [23]). Finally such a relation is also supported by the observation in ref. [20], that the result in the Good-Walker formalism indeed has the expected triple-pomeron form.

In order to illuminate the relation between the two formalisms, we first study a simple toy model. We will later discuss the relation between the toy model and the real QCD evolution. The toy model was proposed by Mueller to illustrate effects of multiple scattering and saturation [24, but here we will instead use it in the weak interaction limit, to demonstrate the similarities between the triple-pomeron and Good-Walker formalisms for high-mass diffraction. The model has the same basic structure as onium-onium scattering in 4-dimensional QCD, but is made simpler by eliminating the transverse degrees of freedom. Thus, although it is not an approximation to QCD in 4 dimensions, it does have the same basic structure as the BFKL pomeron.

Also other approaches to a description of gap events have been presented. In the "color reconnection" model it is assumed that the color exchange in a "normal" inelastic event can be neutralized through the mediation of soft gluons [25, 26], and in the Ingelman-Schlein picture events in diffractive excitation are described in terms of a pomeron flux factor and parton distribution functions in the pomeron [27]. For these approaches the relation to diffraction in optics is not clear, and they will not be discussed here.

\section{The toy model}

The toy model has a structure with essential similarities to BFKL evolution in the dipole formulation of QCD. In the model there is only one type of dipole (or parton), which evolves into a cascade when the rapidity $y$ is increased. A dipole can emit a new dipole with a probability $\alpha$ per unit rapidity. We let $P_{n}(y)$ denote the probability for the cascade to contain $n$ dipoles at rapidity $y$. This distribution satisfies the following evolution equation

$$
\frac{d P_{n}}{d y}=\alpha\left[(n-1) P_{n-1}-n P_{n}\right] .
$$

Here the first term describes the gain when a new dipole is emitted in a state with $(n-1)$ dipoles, and the second term the loss when a dipole emission changes a state with $n$ dipoles into a state with $(n+1)$ dipoles. With the boundary value $P_{n}(0)=\delta_{n 1}$, the solution to this equation is

$$
P_{n}(y)=e^{-\alpha y}\left(1-e^{-\alpha y}\right)^{n-1} .
$$

The average and variance of this multiplicity distribution are given by

$$
\begin{aligned}
\langle n\rangle & =e^{\alpha y} \\
V & \equiv\left\langle n^{2}\right\rangle-\langle n\rangle^{2}=e^{2 \alpha y}-e^{\alpha y} .
\end{aligned}
$$


We note that for asymptotic energies (large $y$ ) the distribution satisfies KNO scaling

$$
P_{n} \approx \frac{1}{\langle n\rangle} F(n /\langle n\rangle), \text { with } F(\xi)=e^{-\xi} .
$$

This approximation would give $V=e^{2 \alpha y}$, and the second term in eq. (2.4) is a result of the deviation from exact scaling for finite (and discrete) $n$-values.

When two dipoles meet, they interact inelastically with probability $2 \alpha^{2} f$, and in the weak interaction limit the optical theorem gives the elastic dipole-dipole scattering amplitude $\alpha^{2} f$. I have here used the same notation as in ref. [24]. In the QCD analogy $\alpha \propto \bar{\alpha}_{s} \equiv N_{c} \alpha_{s} / \pi$ and $f \propto 1 / N_{c}^{2}$.

We study two cascades, evolved in opposite directions distances $y_{1}$ and $y_{2}$ respectively. When they meet the colliding dipoles interact independently, and in the Born approximation the elastic scattering amplitude is given by

$$
T_{\mathrm{el}}=\sum_{n, m} P_{n}\left(y_{1}\right) P_{m}\left(y_{2}\right) n m \alpha^{2} f=\alpha^{2} f e^{\alpha y_{1}} e^{\alpha y_{2}}=\alpha^{2} f e^{\alpha Y}
$$

Here $Y=y_{1}+y_{2}$ denotes the total rapidity range between the projectile and the target, and we note that the result depends only on this total range, and thus is independent of the Lorentz frame used for the calculation. In appropriate units we define $s=e^{Y}$, and thus we have in the weak interaction limit

$$
\begin{aligned}
& \sigma_{\text {el }}=\left(\alpha^{2} f\right)^{2} s^{2 \alpha} \\
& \sigma_{\text {inel }}=2 \alpha^{2} f s^{\alpha},
\end{aligned}
$$

corresponding to a pomeron with intercept $1+\alpha$, and a dipole-pomeron coupling $g_{d P}^{2}=\alpha^{2} f$.

\section{Triple-pomeron formalism}

In the triple-pomeron formalism for diffractive excitation the amplitude is determined by the $3 \rightarrow 3$ scattering process. We study a situation where a parent dipole evolves a distance $y_{1}$, when one of its $n$ daughter dipoles is split in two. These two dipoles interact elastically (and independently) with two separate dipole cascades, both developed a distance $y_{2}$ (see fig. (1). The probability for such a split per unit rapidity is $\alpha$, and in the weak interaction limit the interaction weight for each of the two interactions is $\alpha^{2} f$. With $M_{X}^{2}=e^{y_{1}}$ and $s=e^{y_{1}+y_{2}}$ this gives the cross section for single diffractive excitation

$$
\begin{array}{r}
M_{X}^{2} \frac{d \sigma_{\mathrm{SD}}}{d M_{X}^{2}}=\alpha \sum_{n} P_{n}\left(y_{1}\right) n \sum_{m} P_{m}\left(y_{2}\right) m\left(\alpha^{2} f\right) \sum_{l} P_{l}\left(y_{2}\right) l\left(\alpha^{2} f\right)= \\
=\alpha^{5} f^{2} e^{\alpha y_{1}}\left(e^{\alpha y_{2}}\right)^{2}=\alpha^{5} f^{2}\left(M_{X}^{2}\right)^{\alpha}\left(s / M_{X}^{2}\right)^{2 \alpha} .
\end{array}
$$

This result corresponds to the triple-pomeron expression, where the weight factor $\alpha^{5} f^{2}$ should correspond to $g_{d P}^{3} g_{3 P}$ (with a suitably defined triple-pomeron coupling $g_{3 P}$ ). With $g_{d P}^{2}=\alpha^{2} f$ this gives $g_{3 P}=\alpha^{2} \sqrt{f}$, where a factor $\alpha \sqrt{f}\left(\sim \alpha_{s}\right)$ comes from the interaction weights, and a factor $\alpha\left(\sim \bar{\alpha}_{s}\right)$ from the splitting probability per unit rapidity. 


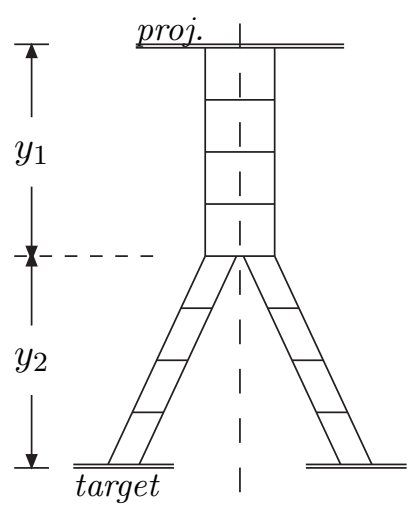

Figure 1: A triple-pomeron diagram

We can in the same way also calculate the total diffractive cross section by replacing the factor $n$ in eq. (3.1) by $n^{2}$. This will include contributions from interaction of two uncorrelated dipoles in the projectile cascade, which corresponds to all possible masses $M_{X}^{2}<M_{X, \max }^{2}=e^{y_{1}}$ (including elastic scattering). The result can be written in the form

$$
\begin{array}{r}
\sigma_{\text {tot proj diff }}=\sum_{n} P_{n}\left(y_{1}\right) n^{2} \sum_{m} P_{m}\left(y_{2}\right) m\left(\alpha^{2} f\right) \sum_{l} P_{l}\left(y_{2}\right) l\left(\alpha^{2} f\right)= \\
=\alpha^{4} f^{2}\left(2 e^{2 \alpha y_{1}}-e^{\alpha y_{1}}\right) e^{2 \alpha y_{2}}=\alpha^{4} f^{2}\left[(s)^{2 \alpha}+(s)^{2 \alpha}\left(1-1 /\left(M_{X, \max }^{2}\right)^{\alpha}\right)\right] .
\end{array}
$$

Here the first term in the square parenthesis corresponds to the elastic cross section from eq. (2.7). The second term represents the integrated sum over diffractive masses in eq. (3.1), given by the single diffractive excitation cross section

$$
\sigma_{\mathrm{SD}}=\int_{0}^{y_{1}} d y_{1} \alpha^{5} f^{2} e^{2 \alpha Y} e^{-\alpha y_{1}}=\alpha^{4} f^{2} e^{2 \alpha Y}\left(1-e^{-\alpha y_{1}}\right)
$$

Subtracting the elastic cross section, given by the square of the amplitude in eq. (2.6), from the result in eq. (3.2), we note that the diffractive excitation also can be written in the form

$$
\sigma_{\mathrm{SD}}=\left\{\sum_{n} P_{n}\left(y_{1}\right) n^{2}-\left(P_{n}\left(y_{1}\right) n\right)^{2}\right\}\left(\sum_{m} P_{m}\left(y_{2}\right) m\left(\alpha^{2} f\right)\right)^{2} .
$$

Thus we see that the result is actually determined by the fluctuations in the multiplicity distribution $P_{n}\left(y_{1}\right)$, given by the variance $\left\langle n^{2}\right\rangle-\langle n\rangle^{2}$.

\section{Good-Walker formalism}

In the Good-Walker formalism the mass eigenstates $\Psi_{k}$ are written as linear combinations of the diffractive eigenstates $\Phi_{i}, \Psi_{k}=\sum c_{k i} \Phi_{i}$, where $c_{k i}$ is a unitary matrix. For scattering against a structureless target, and with $\Psi_{\text {in }}=\Psi_{0}$, the elastic amplitude is given by the average over the diffractive eigenstates:

$$
\left\langle\Psi_{0}|T| \Psi_{0}\right\rangle=\sum\left|c_{0 i}\right|^{2} T_{i}=\langle T\rangle .
$$


The amplitude for diffractive transition to the mass eigenstate $\Psi_{k}$ becomes

$$
\left\langle\Psi_{k}|T| \Psi_{0}\right\rangle=\sum_{i} c_{i k}^{*} T_{i} c_{0 i}
$$

Neglecting the transverse coordinates this gives a total diffractive cross section

$$
\sigma_{\text {diff }}=\sum_{k}\left\langle\Psi_{0}|T| \Psi_{k}\right\rangle\left\langle\Psi_{k}|T| \Psi_{0}\right\rangle=\left\langle T^{2}\right\rangle
$$

and after subtracting the elastic scattering, the cross section for diffractive excitation is given by the fluctuations:

$$
\sigma_{\text {diff exc }}=\left\langle T^{2}\right\rangle-\langle T\rangle^{2} \equiv V_{T},
$$

For scattering of two dipole cascades, the cross section for diffractive scattering of the projectile (including elastic), and elastic scattering of the target, is given by $\left\langle\langle T\rangle_{\operatorname{targ}}^{2}\right\rangle_{\text {proj }}$. In the toy model the cross section, $\sigma_{S D}$, for diffractive excitation of the projectile, and elastic scattering of the target, is thus given by

$$
\begin{aligned}
\sigma_{\mathrm{SD}} & =\sigma_{\text {tot proj diff }}-\sigma_{\mathrm{el}}=\left\langle\langle T\rangle_{\text {targ }}^{2}\right\rangle_{\text {proj }}-\left(\left\langle\langle T\rangle_{\text {targ }}\right\rangle_{\text {proj }}\right)^{2}= \\
& =\sum_{n} P_{n}\left(y_{1}\right) n^{2}\left(\sum_{m} P_{m}\left(y_{2}\right) m \alpha^{2} f\right)^{2}-\left(\sum_{n} P_{n}\left(y_{1}\right) n \sum_{m} P_{m}\left(y_{2}\right) m \alpha^{2} f\right)^{2}= \\
& =\left(\alpha^{2} f\right)^{2} e^{2 \alpha Y}\left(1-e^{-\alpha y_{1}}\right) .
\end{aligned}
$$

This cross section includes all excited systems $X$ confined within the rapidity range $0<y<$ $y_{1}$, and the average over target and projectile states correspond to $\sum_{m} P_{m}$ and $\sum_{n} P_{n}$ respectively. We see that the result exactly reproduces the triple-pomeron result in eqs. (3.4, 3.3); in both formalisms the cross section is determined by the fluctuations in the evolution. Taking the derivative with respect to $y_{1}$, we get also in the Good-Walker formalism the differential result in eq. (3.1).

\section{Relation to QCD}

We have seen above that in the toy model the dipole splitting probability per unit rapidity, $\alpha$, determines the fluctuations in the evolution in eq. (2.4), and also the differential diffractive cross section in eq. (3.1) via the factor $\alpha \sum_{n} P_{n}\left(y_{1}\right) n$. Analogous results are obtained for real QCD in three space dimensions by Iancu and Triantafyllopoulos in [22], which show that the dipole splitting term determines both the fluctuations in the evolution and the triple-pomeron coupling. In their notation the probability per unit rapidity for a dipole $(\mathbf{x}, \mathbf{y})$ to split into two connected dipoles, $(\mathbf{x}, \mathbf{z})$ and $(\mathbf{z}, \mathbf{y})$, (sharing a common gluon) is given by

$$
\frac{\bar{\alpha}_{s}}{2 \pi} \mathcal{M}(\mathbf{x}, \mathbf{y}, \mathbf{z})=\frac{\bar{\alpha}_{s}}{2 \pi} \frac{(\mathbf{x}-\mathbf{y})^{2}}{(\mathbf{x}-\mathbf{z})^{2}(\mathbf{z}-\mathbf{y})^{2}} .
$$

The dipole density after evolution $y$ units of rapidity, is denoted $n_{y}(\mathbf{x}, \mathbf{y})$, and the dipoledipole scattering cross section $\mathcal{A}_{0}(\mathbf{x}, \mathbf{y} \mid \mathbf{u}, \mathbf{v})$. With these notations the differential diffractive cross section can be extracted from their eq. (5.5), and be expressed in the following 
form (the integral sign indicates appropriate integrations over transverse coordinates)

$M_{X}^{2} \frac{d \sigma_{\mathrm{SD}}}{d M_{X}^{2}} \propto \int \bar{\alpha}_{s} \mathcal{M}(\mathbf{u}, \mathbf{v}, \mathbf{z}) n_{y_{1}}(\mathbf{u}, \mathbf{v}) n_{y_{2}}\left(\mathbf{x}_{\mathbf{1}}, \mathbf{y}_{\mathbf{1}}\right) \alpha_{s}^{2} \mathcal{A}_{0}\left(\mathbf{x}_{\mathbf{1}}, \mathbf{y}_{\mathbf{1}} \mid \mathbf{u}, \mathbf{z}\right) n_{y_{2}}\left(\mathbf{x}_{\mathbf{2}}, \mathbf{y}_{\mathbf{2}}\right) \alpha_{s}^{2} \mathcal{A}_{0}\left(\mathbf{x}_{\mathbf{2}}, \mathbf{y} \mathbf{2} \mid \mathbf{z}, \mathbf{v}\right)$.

With the translation $\bar{\alpha}_{s} \mathcal{M} \rightarrow \alpha$ and $\alpha_{s}^{2} \mathcal{A}_{0} \rightarrow \alpha^{2} f$, this result exactly corresponds to the toy model expression in eq. (3.1). Thus we conclude that the relation between the triplepomeron and Good-Walker formalisms should hold also in real QCD. Important for the result is also the fact, that these fluctuations are large, and lead to approximate KNO scaling, which is a basic feature of QCD evolution (see e.g. ref. [29]).

It ought to be noticed that in LL BFKL evolution the pomeron singularity is a cut, which implies that the triple-pomeron coupling obtained from the integral over $\mathcal{M}$ in eq. (5.2) will depend on the energy. It is, however, well known that with a running coupling the cut is replaced by a series of poles [28]. For high energies the leading pole is dominating, and if the corresponding eigenfunction to the evolution operator is denoted $\phi(\mathbf{x}-\mathbf{y})$, the triple-pomeron coupling is given by the energy independent expression

$$
g_{3 P} \propto \int \phi(\mathbf{x}-\mathbf{z}) \phi(\mathbf{z}-\mathbf{y}) \bar{\alpha} \mathcal{M}(\mathbf{x}, \mathbf{y}, \mathbf{z}) \phi(\mathbf{x}-\mathbf{y}) d^{2} x d^{2} z .
$$

The connection between the triple-pomeron and Good-Walker formalisms is also reflected in the results from the dipole cascade model DIPSY, which is based on BFKL evolution and the Good-Walker picture for diffractive excitation. These results indeed reproduce the expected triple-pomeron form, with a simple pomeron pole and an approximately constant triple-pomeron coupling, once the non-linear effects are switched off [20]. ${ }^{1}$

\section{Discussion}

Essential for the results described above is that in both the triple-pomeron and GoodWalker formalisms, the diffractive excitation is determined by the fluctuations. We also note that it is the small correction term in the expression for the variance in eq. (2.4), which is responsible for the differential diffractive cross section in eq. 3.1). At high energies (and disregarding saturation effects) the integrated single diffractive cross section saturates at $\sigma_{\mathrm{SD}}=\left(\alpha^{2} f\right)^{2} e^{2 \alpha Y}$, given by the scaling part in the variance $\propto e^{2 \alpha y}$ in eq. (2.4). From the fact that $d \sigma_{\mathrm{SD}} / d \ln \left(M_{X}^{2}\right)$ drops for large $M_{X}$ at constant $s$, one could possibly get the impression that the fluctuations are less important in this case. This is, however, not true. For a fixed target system with constant $y_{2}$, the single diffractive cross section would grow $\sim e^{2 \alpha y_{1}}=\left(M_{X, \max }^{2}\right)^{2 \alpha}$. For fixed $s=e^{\left(y_{1}+y_{2}\right)}$ the increased fluctuations in the projectile cascade for larger $y_{1}\left(\propto\left(e^{2 \alpha y_{1}}-e^{\alpha y_{1}}\right)\right)$ are compensated by an approximately equally fast decrease in the target evolution $\propto\left(e^{\alpha y_{2}}\right)^{2}$. The suppressed term $-e^{\alpha y_{1}}$ in the variance in eq. (2.4) implies that the differential diffractive excitation $d \sigma_{\mathrm{SD}} / d y_{1}$ is proportional to $\alpha e^{-\alpha y_{1}}=\alpha /\left(M_{X}^{2}\right)^{\alpha}$, over a range of the order $1 / \alpha$ in $y_{1}=\ln \left(M_{X}^{2}\right)$.

\footnotetext{
${ }^{1}$ In perturbative QCD with massless gluons and without confinement, the triple-pomeron coupling has a singularity $\sim 1 / \sqrt{-t}[30,31$. In the DIPSY model confinement effects are also included (they are also important for satisfying the Froissart-Martin bound at asymptotic energies [32]). Also in recent triple-regge fits by the Tel Aviv [15] and Durham 13 groups, the results correspond to a simple pomeron pole.
} 
The Good-Walker formalism has the advantage that it is highly predictive. Including saturation effects (related to enhanced and semienhanced diagrams) the DIPSY model, based on the Good-Walker formalism, reproduces experimental results for diffraction in DIS and $p p$ collisions, without extra tunable parameters besides those tuned to the total and elastic cross sections [19, 20, 21]. Thus e.g. the triple-pomeron coupling is fully determined by the dynamics via the optical theorem. This is in contrast to phenomenological applications of the triple-regge formalism, where the triple-pomeron coupling is an adjustable parameter, which has to be fitted to data.

In the discussions above we have only discussed pomeron exchange, which dominates for high energies and excitation masses, $s>>M_{X}^{2}>>\Lambda_{\mathrm{QCD}}$. For lower energy or excitation mass, also lower regge trajectories contribute. These contributions are not included in the DIPSY dipole model. Nevertheless, with a cutoff for low masses, $M_{X}$, of the order of 1 $\mathrm{GeV}$, the results are consistent with data from refs. [1]- [5]. (As far as we know, no model is able to reproduce the full resonance structure of low mass excitations.)

\section{Conclusion}

In conclusion we argue that the dynamics of the BFKL pomeron implies that the triplepomeron and the Good-Walker formalisms for high mass diffraction are just different formulations of the same phenomenon. In both formalisms diffractive excitation is the shadow of absorption into inelastic channels, and the similarity is related to the KNO scaling in QCD evolutions, where the fluctuations in the gluon multiplicity grow proportional to the multiplicity, $\sigma^{2} \approx\langle n\rangle^{2}$. A phenomenological analysis based on the Good-Walker formalism has the advantage that diffractive excitation is fully determined by the dynamics via the optical theorem, with no extra parameters beyond those tuned to the total and elastic cross sections.

The relation between the two formalisms is here illustrated by an analysis of a simple toy model in one plus one dimensions, but we also see that the essential features of this model are shared by BFKL evolution in real QCD in three space dimensions. The relation is further supported by results from the Lund dipole cascade model DIPSY, based on BFKL evolution and using the Good-Walker formalism. Excluding non-linear effects, the model reproduces the triple-pomeron form for diffractive excitation [20], and including also effects of saturation it reproduced both inclusive and exclusive diffractive data in DIS and $p p$ collisions [19, 20, 21.

\section{Acknowledgments}

The author is grateful to Leif Lönnblad for valuable discussions. I also want to thank the referee for pointing out the resemblence between the toy model and the results in refs. [22, 23].

\section{References}

[1] H1 Collaboration, C. Adloff et al., Z. Phys. C76 (1997) 613-629, hep-ex/9708016. 
[2] ZEUS Collaboration, S. Chekanov et al., Nucl. Phys. B713 (2005) 3-80, hep-ex/0501060.

[3] UA4 Collaboration, D. Bernard et al., Phys.Lett. B166 (1986) 459.

[4] UA5 Collaboration, R. E. Ansorge et al., Z. Phys. C33 (1986) 175.

[5] CDF Collaboration, F. Abe et al., Phys. Rev. D50 (1994) 5535-5549.

[6] A. H. Mueller, Phys. Rev. D2 (1970) 2963-2968.

[7] C. E. DeTar et al., Phys. Rev. Lett. 26 (1971) 675-676.

[8] M. L. Good and W. D. Walker, Phys. Rev. 120 (1960) 1857-1860.

[9] V. Abramovsky, V. Gribov, and O. Kancheli, Yad.Fiz. 18 (1973) 595-616.

[10] A. Kaidalov and M. Poghosyan, Eur.Phys.J. C67 (2010) 397-404, arXiv:0910.2050 [hep-ph].

[11] S. Ostapchenko, Phys.Rev. D83 (2011) 014018, arXiv:1010.1869 [hep-ph].

[12] M. Ryskin, A. Martin, and V. Khoze, Eur.Phys.J. C71 (2011) 1617, arXiv:1102.2844 [hep-ph].

[13] M. Ryskin, A. Martin, and V. Khoze, Eur.Phys.J. C72 (2012) 1937, arXiv:1201.6298 [hep-ph].

[14] E. Gotsman, E. Levin, and U. Maor, Eur.Phys.J. C71 (2011) 1553, arXiv:1010.5323 [hep-ph].

[15] E. Gotsman, E. Levin, and U. Maor, Phys.Rev. D85 (2012) 094007, arXiv:1203.2419 [hep-ph].

[16] E. Gotsman, E. Levin, and U. Maor, arXiv:1208.0898 [hep-ph].

[17] H. I. Miettinen and J. Pumplin, Phys. Rev. D18 (1978) 1696.

[18] Y. Hatta, E. Iancu, C. Marquet, G. Soyez, and D. N. Triantafyllopoulos, Nucl. Phys. A773 (2006) 95-155, hep-ph/0601150.

[19] E. Avsar, G. Gustafson, and L. Lonnblad, JHEP 0712 (2007) 012, arXiv:0709.1368 [hep-ph].

[20] C. Flensburg and G. Gustafson, JHEP 1010 (2010) 014, arXiv:1004.5502 [hep-ph].

[21] C. Flensburg, G. Gustafson, and L. Lonnblad, arXiv:1210.2407 [hep-ph].

[22] E. Iancu and D. Triantafyllopoulos, Phys.Lett. B610 (2005) 253-261, arXiv:hep-ph/0501193 [hep-ph].

[23] M. Braun and G. Vacca, Eur.Phys.J. C6 (1999) 147-157, arXiv:hep-ph/9711486 [hep-ph],

[24] A. H. Mueller, Nucl.Phys. B437 (1995) 107-126, arXiv:hep-ph/9408245 [hep-ph].

[25] A. Edin, G. Ingelman, and J. Rathsman, Phys.Lett. B366 (1996) 371-378, arXiv:hep-ph/9508386 [hep-ph].

[26] R. Pasechnik, R. Enberg, and G. Ingelman, Phys.Lett. B695 (2011) 189-193, arXiv:1004.2912 [hep-ph].

[27] G. Ingelman and P. Schlein, Phys.Lett. B152 (1985) 256.

[28] L. N. Lipatov, Sov. Phys. JETP 63 (1986) 904-912. 
[29] E. Malaza and B. Webber, Nucl.Phys. B267 (1986) 702.

[30] A. H. Mueller and B. Patel, Nucl. Phys. B425 (1994) 471-488, hep-ph/9403256.

[31] J. Bartels, M. G. Ryskin, and G. P. Vacca, Eur. Phys. J. C27 (2003) 101-113, arXiv:hep-ph/0207173.

[32] E. Avsar, JHEP 04 (2008) 033, arXiv:0803.0446 [hep-ph]. 